\title{
Improving Type Ia supernova characterization through multiwavelength studies of Type Ia supernova host galaxies
}

\author{
Brad Tucker, ${ }^{1}$ Brian P. Schmidt,${ }^{1}$ and Peter Garnavich ${ }^{2}$ \\ ${ }^{1}$ Research School of Astronomy and Astrophysics, Australian National University, Canberra, \\ Australia \\ email: brad@mso.anu.edu. au \\ ${ }^{2}$ Department of Physics, University of Notre Dame, 225 Nieuwland Science Hall, Notre Dame, \\ IN 46556, USA
}

\begin{abstract}
Past investigations have shown a connection between the properties of Type Ia supernovae (SNe Ia) and their host galaxies. We refine these studies using ultraviolet through midinfrared observations of both nearby and distant SN Ia hosts. We present new results showing that the properties of SNe Ia, both intrinsic and with respect to their use as distance indicators, appear to depend on a combination of metallicity, stellar age, and star-formation rate of the host. We suggest that the stellar population age and location of the supernova progenitor all can play a roll in using SNe Ia as precision distance indicators, and advocate that a multiwavelength approach is one way to disentangle the different influences, resulting in an improvement of $8 \%$ in distance measurements.
\end{abstract}

Keywords. supernovae: general, galaxies: distances and redshifts, distance scale 